\title{
The effect of Schroth's three-dimensional exercises in combination with respiratory muscle exercise on Cobb's angle and pulmonary function in patients with idiopathic scoliosis
}

\author{
Min-Jae Kim, Dae-Sung Park \\ Department of Physical Therapy, College of Medical Science, Konyang University, Daejeon, Republic of Korea
}

Objective: The objective of this study was to investigate the effects of Schroth's three-dimensional exercises in combination with respiratory muscle exercise (SERME) on Cobb's angle and functional movement screen (FMS).

Design: Randomized controlled trial.

Methods: Fifteen subjects with scoliosis were randomly assigned to two groups. Eight subjects were assigned to the experimental group and seven subjects were assigned to the control group. The experimental group underwent SERME using SpiroTiger (Idiag, Switzerland), while the control group performed only the Schroth's three-dimensional exercises (SE). Both groups performed exercises for one hour per day, three times a week for eight weeks. Cobb's angle, pulmonary function (forced vital capacity, forced expiratory volume at one second, and peak expiratory flow) and FMS were measured before and after the experiment.

Results: After intervention, the SERME group showed a significant difference in Cobb's angle, FMS scores, and pulmonary function as compared to before intervention $(p<0.05)$. In the SE group, there was a statistically significant difference in Cobb's angle, pulmonary function, and FMS scores compared to before intervention $(p<0.05)$. The SERME group showed a significant difference in Cobb's angle and peak expiratory flow in pulmonary function compared to the SE group $(p<0.05)$.

Conclusions: The results suggest that SERME could be a more effective intervention for improvement of the Cobb's angle and pulmonary function for scoliosis patients.

Key Words: Breathing exercises, Pulmonary ventilation, Scoliosis

\section{Introduction}

Without proper treatment, scoliosis could lead to functional deterioration led by asymmetry of the body, muscle imbalance, back pain and severe respiratory disturbance, resulting in the deformation of body image along with loss of self-esteem. In particular, since the rib is connected to the vertebral body, the rotation of the vertebral body could cause the convex surface of the curvature to form the hump on the posterior trunk while the curved concave surface forms the hump on the anterior trunk. Scoliosis causes an interference with respiration and asymmetric breathing occurs on the convex and concave surfaces. The deformation of the trunk limits the mobility of the thorax, which in turn, reduces the volume of breathing!

As for general treatments of scoliosis, manual therapy and therapies using slings and balls [1,2] are available. The Schroth's three-dimensional exercises (SE), developed by Katharina Schorth in Germany, is considered to be more effective that these manual treatments because they are three-dimensional (3D) [3-5]. SE requires a patient with scoliosis to recognize $3 \mathrm{D}$ changes shown in the patient, and

Received: 26 August, 2017 Revised: 14 September, 2017 Accepted: 14 September, 2017

Corresponding author: Dae-Sung Park

Department of Physical Therapy, College of Medical Science, Konyang University, 158 Gwanjeodong-ro, Seo-gu, Daejeon 35365, Republic of Korea Tel: 82-42-600-6419 Fax: 82-42-600-6419 E-mail: daeric@naver.com

(c) This is an Open-Access article distributed under the terms of the Creative Commons Attribution Non-Commercial License (http://creativecommons.org/licens es/by-nc/4.0) which permits unrestricted non-commercial use, distribution, and reproduction in any medium, provided the original work is properly cited.

Copyright $@ 2017$ Korean Academy of Physical Therapy Rehabilitation Science 
restores the spinal structure and function by correcting the trunk. The most significant factor of this exercise, differentiating it from other therapies, is that it consists of rotational angular breathing (RAB), which uses the rotation of the deformed rib with breathing [5].

In the previous studies on SE, it was reported that after seven days of the new power Schroth's exercise, there was a significant decrease in the Cobb's angle from $28.7^{\circ}$ to $13.7^{\circ}$ [6]. Also, after 6 months of SE for a girl with scoliosis, the Cobb's angle was reduced from $52^{\circ}$ to $42^{\circ}$ and the rotation angle of the trunk was decreased from $14^{\circ}$ to $10^{\circ}$ [7]. For patients with scoliosis, breathing expanding the thorax and diaphragmatic breathing corrects the pattern of breathing and increases the mobility of the thorax and functions of the lungs [8].

Functional movement screen (FMS) determines the extent of joint motions, flexibility, strength, balance, coordination, and proprioceptive sensation and it is constructed with seven standard functional movements. The primary purpose of the FMS is to test an individual's ability to perform essential body movements.

The previous studies on scoliosis have focused on static postures and there are only limited numbers of studies on functional postures, such as the SE. Therefore, this study aims to investigate the effects of the Schroth's three-dimensional exercises in combination with respiratory muscle exercise (SERME) on Cobb's angle, pulmonary function, and functional movements in persons with scoliosis.

\section{Methods}

\section{Participants}

As for subjects of this study, 15 patients diagnosed with scoliosis with a Cobb's angle of $10^{\circ}[9,10]$ or above indicated by orthopedic surgeons of hospital $\mathrm{N}$, located in Daegu were selected as candidates. The selection criteria of the participants were as follows: patients with a Cobb's angle of $10^{\circ}$ or above, no treatment experience for the last three months, and no orthopedic problems other than scoliosis. The exclusion criteria were as follows: patients with asthma or other pulmonary diseases, central nervous system disease, or history of spinal surgery. All subjects were suitably notified of the testing process and signed the research agreement. Approval from the Konyang University Institutional Review Board (IRB No. 2016-034) was obtained.

\section{Experimental procedures}

In this study, a preliminary interview was conducted with the questionnaire to evaluate the general characteristics. Eight participants were randomly assigned to a group administering SERME and seven participants were assigned to the other group administering only SE. As for the treatment group applied SERME. The Cobb's angle, pulmonary function and FMS were measured before and after 8 weeks of the exercise program for one hour per day, three times a week.

A preparatory exercise was conducted for 5 minutes followed by respiratory muscle exercise (RME) that was performed using the Spiro tiger for 10 minutes. Afterwards, SE was conducted for 40 minutes. The control group underwent a preparatory exercise for five minutes, followed by exercising on a fixed bicycle for 10 minutes, performed only the SE for 40 minutes, and then concluded with a five-minute finishing exercise. The group prescribed with SERME performed the preparation exercise for five minutes before the exercise and then, the RME in the sitting position for $10 \mathrm{mi}-$ nutes, followed by SE for 40 minutes and the finishing exercise for five minutes. In the SE group, the five-minute preparation exercise, 10-minute fixed bicycle exercise, 40-minute SE, and five minutes finishing exercise were performed (Tables 1, 2).

The exercise was performed using SE designed in accordance with the curvature of the individuals, and was performed with the 3D RAB of SE while performing each exercise. The self-correction exercise was given so the patient could reconstruct the 3D spinal movement independently [11]. The RME was performed in a sitting posture for two to three times before starting the exercise to allow the patient to understand the procedure and then, it was performed in two sets, with five minutes for each set. Respiration was performed by expanding the concave part of the scoliosis curve at the time of inspiration and shrunken at the convex part of the curve at the time of exhalation.

The SE was categorized into two types: thoracic type and lumbar type. In the case of the thoracic type, subjects were requested to exercise while performing $\mathrm{RAB}$ in which the concave surface of the thoracic vertebra was depressed for inhalation and the convex surface was pushed outwards in three directions (ventral, cranial, and medial). On the other hand, in the case of the lumbar type, subjects were required to perform $\mathrm{RAB}$ in which the concave surface of the lumbar vertebra was used for inhaling and the convex surface was pushed outwards in three-dimensions (ventral, cranial, and 
Table 1. Method of Schroth's three-dimensional exercises

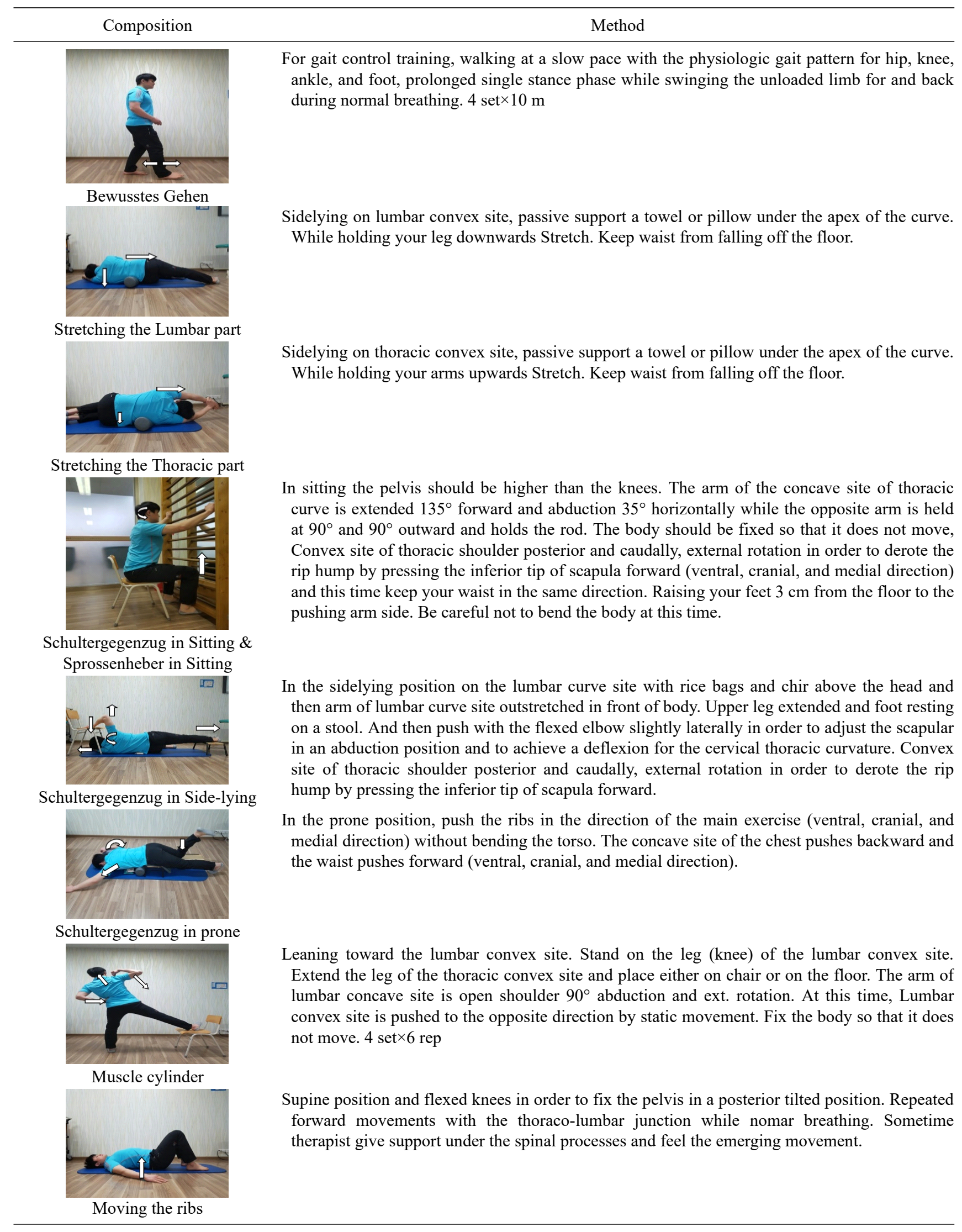


Table 2. Schroth's three-dimensional exercises program

\begin{tabular}{lll}
\hline \multicolumn{1}{c}{ Exercise sequence } & Schroth's three-dimensional exercises program & \multicolumn{1}{c}{ Frequency } \\
\hline Warm-up $(15 \mathrm{~min})$ & Bewusstes gehen & $4 \mathrm{set} \times 10 \mathrm{~m}$ \\
& Respiratory muscle exercise (Spirotiger or Cycle) & $10 \mathrm{~min}$ \\
Stretching \& exercise $(40 \mathrm{~min})$ & Stretching the lumbar part & $10 \mathrm{sec} \times 15 \mathrm{time}$ \\
& Stretching the thoracic part & $10 \mathrm{sec} \times 15 \mathrm{time}$ \\
& Schultergegenzug in sitting & $4 \operatorname{set} \times 6 \mathrm{rep}$ \\
& Sprossenheber in sitting & $4 \operatorname{set} \times 6$ rep \\
& Schultergegenzug in side-lying & $4 \operatorname{set} \times 6$ rep \\
Cool down $(5 \mathrm{~min})$ & Schultergegenzug in prone & $4 \operatorname{set} \times 6$ rep \\
& Muscle cylinder & $4 \operatorname{set} \times 6$ rep \\
\hline
\end{tabular}

medial) during the exercise.

The RME was performed using the SpiroTiger (Idiag, Fehraltorf, Switzerland). For the exercise, the patient was sitting on a chair with the mouthpiece in the mouth and looking at the main body of the SpiroTiger (Idiag). When the therapist pressed the start button, the orange scale of the main body of the device began to move and when it pointed 'In,' the patient was instructed to inhale and when it pointed 'Out,' the patient was instructed to exhale. The therapist informed the patient that the orange sign should change to green and a beep sound should be given when the breathing is performed properly (Figure 1).

\section{Outcome measurement}

\section{Cobb's angle}

Cobb's angle was measured with a radiograph. Radiographs were taken on the full spine at AP view by a radiological technologist with the knees together, the waist and chest extended, the body weight evenly spread onto both feet, and both arms raised while holding one's breath after exhaling a bit. The thoracic or lumbar angles were measured using the Cobb's angle measurement tool stored in the computer. Measurements were made on the Cobb's angle, the angle formed between a line drawn parallel to the superior endplate of one vertebra and a line drawn parallel to the inferior endplate of the vertebra [12].

\section{Pulmonary function measurement}

The measurements of pulmonary functions were taken in a sitting position using CardioTouch 3000S (Bionet, Seoul, Korea). Before commencing the measurement, the patient was requested perform normal breathing for three times, and then to inhale the air as much as possible, and then to exhale as fast as possible to measure the maximal-effort expiratory

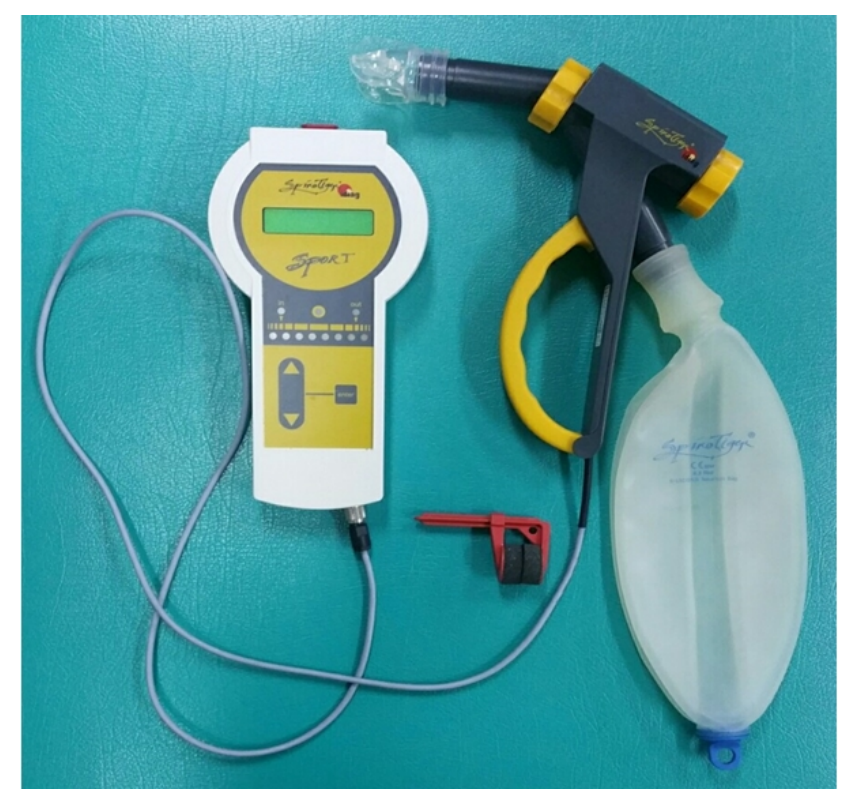

Figure 1. Respiratory muscle exercise.

spirogram. With the maximal-effort expiratory spirogram, the forced vital capacity (FVC), forced expiratory volume at one second $\left(\mathrm{FEV}_{1}\right)$, and the ratio between $\mathrm{FEV}_{1}$ and $\mathrm{FVC}$ was obtained. In addition, airway resistance was measured using the peak expiratory flow (PEF).

\section{Functional movement screen}

FMS was used to evaluate the quality of functional movements. It is used to evaluate seven movements with the lowest score of one and highest score of three points given if the individual experiences pain. The total score possible is 21 points. The subscale of the FMS consists of a deep squat movement pattern, hurdle step movement pattern, inline lunge movement pattern, shoulder mobility reaching movement pattern, active straight leg raise movement pattern, 
trunk stability pushup movement pattern, and rotary stability movement pattern. FMS had a good intra-rater reliability $(\mathrm{ICC}=0.6)$ [13].

\section{Statistical analysis}

All statistical analyses were performed using IBM SPSS Statistics ver. 18.0 (IBM Co., Armonk, NY, USA). As for general characteristics of the subjects, descriptive statistics were used. For all dependent variables, normal distribution of data was confirmed by the Shapiro-Wilks test. The comparison of the participants before and after the experiment in terms of the Cobb's angle, pulmonary function and FMS changes was conducted with the paired t-test, and the independent $t$-test was conducted for comparative analysis of the test group and the control group for the Cobb's angle and FMS scores. The statistical significance was assumed to be $\alpha=0.05$.

\section{Results}

The general characteristics of subjects of this study were as follows. There were no significant differences in the age, height, weight, and Cobb's angle between the SERME and SE groups (Table 3). In the SERME group, there was a significant difference in Cobb's angle, FMS scores and pulmonary functions after intervention $(p<0.05)$. In the SE group, there was a significant difference in Cobb's angle, pulmonary functions and FMS score after intervention $(p<0.05)$. The SERME group showed a significant difference in Cobb's angle and peak expiratory flow in pulmonary functions compared to SE group ( $p<0.05$; Table 4).

Table 3. General characteristics $(\mathrm{N}=15)$

\begin{tabular}{lccr}
\hline \multicolumn{1}{c}{ Characteristic } & $\begin{array}{c}\text { SERME group } \\
(\mathrm{n}=8)\end{array}$ & $\begin{array}{c}\text { SE group } \\
(\mathrm{n}=7)\end{array}$ & $\mathrm{t}(p)$ \\
\hline $\begin{array}{l}\text { Gender } \\
\quad \text { female/male) }\end{array}$ & $6 / 2$ & $4 / 3$ & - \\
Age $(\mathrm{yr})$ & $17.75(4.71)$ & $15.57(2.70)$ & $1.075(0.30)$ \\
Height $(\mathrm{cm})$ & $164.38(8.90)$ & $162.71(2.93)$ & $0.498(0.63)$ \\
Weight $(\mathrm{kg})$ & $50.75(7.96)$ & $52.43(8.38)$ & $-0.398(0.70)$ \\
Cobb's angle $\left(^{\circ}\right)$ & $24.49(8.32)$ & $27.16(12.44)$ & $-0.495(0.63)$ \\
\hline
\end{tabular}

Values are presented as mean (SD).

SERME: Schroth's three-dimensional exercises in combination with respiratory muscle exercise, SE: Schroth's three-dimensional exercise.

\section{Discussion}

In this study, 15 patients with idiopathic scoliosis with Cobb's angle of $10^{\circ}$ or above have participated. They were examined for any changes shown on the Cobb's angle, pulmonary function $\left(\mathrm{FVC}, \mathrm{FEV}_{1}, \mathrm{FEV}_{1} / \mathrm{FVC}\right.$, and PEF) and FMS scores.

In the SERME group, there were significant changes in FVC, $\mathrm{FEV}_{1}$, and PEF before and after exercise. Also, the intergroup analysis has shown that there were significant differences in PEF between before and after the exercise. Changes in posture may affect cardiovascular, digestive, respiratory, and musculoskeletal systems and should therefore be considered as important in medical management and

Table 4. Comparative analysis of Cobb's angle, functional movement screen and pulmonary functions $(\mathrm{N}=15)$

\begin{tabular}{lccr}
\hline Characteristic & $\begin{array}{c}\text { SERME group } \\
(\mathrm{n}=8)\end{array}$ & $\begin{array}{c}\text { SE group } \\
(\mathrm{n}=7)\end{array}$ & $\mathrm{t}$ \\
\hline Cobb's angle $\left(^{\circ}\right)$ & & & \\
Pre & $24.49(8.32)$ & $27.16(12.44)$ & -0.495 \\
Post & $20.23(7.54)$ & $24.47(12.45)$ & -0.810 \\
Difference & $4.26\left(1.36^{*}\right)$ & $2.69\left(1.11^{*}\right)$ & $2.415^{*}$ \\
Functional movement screen (score) & & \\
Pre & $10.25(1.91)$ & $11.14(2.48)$ & -0.788 \\
Post & $15.50(1.31)$ & $14.43(1.90)$ & 1.286 \\
Difference & $-5.25\left(1.83^{*}\right)$ & $-3.29\left(2.36^{*}\right)$ & -1.814 \\
Pulmonary function & & & \\
FVC (L) & & & \\
Pre & $2.73(0.63)$ & $2.97(0.77)$ & -0.661 \\
Post & $3.06(0.51)$ & $2.75(0.83)$ & 0.872 \\
Difference & $-0.33\left(0.35^{*}\right)$ & $0.21(0.76)$ & -1.818 \\
FEV $(\mathrm{L})$ & & & \\
Pre & $2.32(0.58)$ & $2.38(1.05)$ & -0.162 \\
Post & $2.71(0.41)$ & $2.33(0.83)$ & 1.135 \\
Difference & $-0.39\left(0.30^{*}\right)$ & $0.05(0.64)$ & -1.772 \\
FEV $/$ FVC $(\%)$ & & & \\
Pre & $85.11(9.44)$ & $78.22(26.76)$ & 0.684 \\
Post & $88.87(4.89)$ & $84.64(12.94)$ & 0.861 \\
Difference & $-3.76(13.12)$ & $-6.41(25.09)$ & 0.262 \\
PEF (L) & & & \\
Pre & $3.98(1.49)$ & $4.85(2.89)$ & -0.749 \\
Post & $5.28(0.96)$ & $5.02(2.84)$ & 0.242 \\
Difference & $-1.30\left(0.87^{*}\right)$ & $-0.17(0.68)$ & $-2.769^{*}$ \\
\hline
\end{tabular}

Values are presented as mean (SD).

SERME: Schroth's three-dimensional exercises in combination with respiratory muscle exercise, SE: Schroth's three-dimensional exercise, FVC: forced vital capacity, $\mathrm{FEV}_{1}$ : forced expiratory volume at one second, $\mathrm{FEV}_{1} / \mathrm{FVC}(\%)$ : a ratio between $\mathrm{FEV}_{1}$ and $\mathrm{FVC}$, PEF: peak expiratory flow. $p<0.05$. 
physical therapy [14]. Correction and respiratory therapy should be performed because scoliosis patients have weakened respiratory muscles due to curvature of the thoracic spine, which reduces cardiopulmonary function [15]. Orthodontic treatment and thoracic flexibility enhancement exercises should be performed to remove the compression of the lungs due to deformation and flexibility of the thorax [16]. SE could support the restoration of the respiratory muscles by rotating the twisted rib cage to the opposite direction via the $\mathrm{RAB}[4,5]$, which is considered as an important factor in the improvement of scoliosis. Ottman et al. $[17,18]$ reported that exercise programs that extend the rib cage are useful in increasing pulmonary function and mobility of the ribs. SE and correction exercises decreased Cobb's angle and increased pulmonary function variables (chest expansion and maximal inspiratory pressure) in patients with idiopathic scoliosis, and especially showed significant improvements on the Cobb's angle and pulmonary function in the group administered with SE [19].

The beneficial effect of SE on pulmonary function is somewhat consistent with results of this study. In this study, the SpiroTiger (Idiag) equipment was used to strengthen the respiratory muscle equipment was used for the RME for four weeks for chronic obstructive pulmonary disease patients and an increase in maximum ventilation capacity was reported [20]. The RME has beneficial effects on the ventilation capacity, chest expansion, respiratory strength and endurance [21]. Maximal ventilation and maximal expiratory volume were significantly improved with the RME of six weeks [22], which is consistent with a result of this study. In comparison between the two groups, significant increase improvements in the SERME group after the exercise enabling a stronger and longer respiration which in turn, reduced the Cobb's angle and improved the pulmonary function.

In this study, the pre and post exercise FMS scores were significantly different in both the SERME and SE group. In a previous study on FMS, a significant improvement in FMS scores was reported after conducting corrective exercise in athletes [23]. The ability to perform was enhanced more in athletes with a FMS score of 14 or higher than those with a lower FMS score [24]. The risk of injury was higher if the FMS score was below 14 points [25]. In addition, there is a correlation between respiratory pattern abnormality and FMS scores, and those with respiratory problems reported low FMS scores [26]. FMS is constructed with fundamental movements to detect the motor control ability of funda- mental movements and actions. While making a FMS movement, the limitation, asymmetry and defects of such movement should be monitored and then, linked with its results. More precise measurements can be used if necessary, but its primary objective is to roughly detect major asymmetry or defect. The original idea of this test was to assess the qualitative aspects of a movement with a simple scoring system, rather than diagnosing and measuring fixed joint movements. In this study, the post-exercise FMS score was significantly increased in the SERME and SE group, which could be led by the improvement balance of the trunk through exercise.

This study has several limitations. First, since the number of participants was limited, it would be rather impractical to generalize the findings of this study. Also, since a short-term intervention was used in this study, it would be necessary to have the follow-up to monitor the long-term effects of the given exercise. Therefore, further studies would be necessary with long-term intervention of the SERME in a larger group of scoliosis patients.

In this study, it was possible to validate the therapeutic effects of SE and the concurrent administration of SERME, but there was a greater effect shown in the SERME group, showing that the SERME group is more effective than SE. In other words, both exercise therapies could produce a significant therapeutic effect in patients with scoliosis in terms of Cobb's angle, but the concurrent administration of SERME could be more effective for patients with spinal scoliosis in terms of pulmonary function and FMS.

\section{Conflict of Interest}

The authors declared no potential conflicts of interest with respect to the authorship and/or publication of this article.

\section{References}

1. Pugacheva N. Corrective exercises in multimodality therapy of idiopathic scoliosis in children-analysis of six weeks efficiencypilot study. Stud Health Technol Inform 2012;176:365-71.

2. Lee JH, Kim SY. Comparative effectiveness of Schroth therapeutic exercise versus sling therapeutic exercise in flexibility, balance, spine angle and chest expansion in patient with scoliosis. J Korean Soc Phys Med 2014;9:11-23.

3. Negrini S, Atanasio S, Zaina F, Romano M. Rehabilitation of adolescent idiopathic scoliosis: results of exercises and bracing from a series of clinical studies. Europa Medicophysica-SIMFER 2007 Award Winner. Eur J Phys Rehabil Med 2008;44:169-76. 
4. Lehnert-Schroth C. The "four-curved" scoliosis. The effect on the additional lumbosacral spinal curve by the three dimensional Schroth's scoliosis treatment. ZFA (Stuttgart) 1981;57:2227-31.

5. Lehnert-Schroth C. Schroth's three dimensional treatment of scoliosis. ZFA (Stuttgart) 1979;55:1969-76.

6. Borysov M, Borysov A. Scoliosis short-term rehabilitation (SSTR) according to 'Best Practice' standards-are the results repeatable? Scoliosis 2012;7:1.

7. Herling O. Clinical measurements, radiological and cosmetic improvements in a girl with adolescent idiopathic scoliosis treated with a Schroth rehabilitation program: a single case study. Scoliosis 2009;4(Suppl 2):O28.

8. Weiss HR. The effect of an exercise program on vital capacity and rib mobility in patients with idiopathic scoliosis. Spine (Phila Pa 1976) 1991;16:88-93.

9. Reamy BV, Slakey JB. Adolescent idiopathic scoliosis: review and current concepts. Am Fam Physician 2001;64:111-6.

10. Kuru T, Yeldan İ, Dereli EE, Özdinçler AR, Dikici F, Çolak İ. The efficacy of three-dimensional Schroth exercises in adolescent idiopathic scoliosis: a randomised controlled clinical trial. Clin Rehabil 2016;30:181-90.

11. Fusco C, Zaina F, Atanasio S, Romano M, Negrini A, Negrini S. Physical exercises in the treatment of adolescent idiopathic scoliosis: an updated systematic review. Physiother Theory Pract 2011;27:80-114.

12. Greiner KA. Adolescent idiopathic scoliosis: radiologic decision-making. Am Fam Physician 2002;65:1877-22.

13. Shultz R, Anderson SC, Matheson GO, Marcello B, Besier T. Test-retest and interrater reliability of the functional movement screen. J Athl Train 2013;48:331-6.

14. Gordon S, Jones A, Sealey R, Buettner P. Body position and cardio-respiratory variables in older people. Arch Gerontol Geriatr 2011;52:23-7.

15. Lonstein JE, Winter RB. The Milwaukee brace for the treatment of adolescent idiopathic scoliosis. A review of one thousand and twenty patients. J Bone Joint Surg Am 1994;76:1207-21.

16. Magee DJ. Assessment of posture. In: Magee DJ, editor. Orthopedic physical assessment. 5th ed. St. Louis: Saunders Elsevier;
2002. p. 1017-50.

17. Otman S, Kose N, Yakut Y. The efficacy of Schroth's 3-dimensional exercise therapy in the treatment of adolescent idiopathic scoliosis in Turkey. Saudi Med J 2005;26:1429-35.

18. Otman S, Kose N, Yakut Y. The efficacy of Schroth's 3-dimensional exercise therapy in the treatment of adolescent idiopathic scoliosis in Turkey. Neurosciences (Riyadh) 2005;10:277-83.

19. Park SE, Moon HH, Park YJ. The effect of Schroth treatment and corrective exercise on Cobb's angle and pulmonary function variables of idiopathic scoliosis patients. J Sport Leis Stud 2013;52: 777-88.

20. Bernardi E, Pomidori L, Bassal F, Contoli M, Cogo A. Respiratory muscle training with normocapnic hyperpnea improves ventilatory pattern and thoracoabdominal coordination, and reduces oxygen desaturation during endurance exercise testing in COPD patients. Int J Chron Obstruct Pulmon Dis 2015;10:1899-906.

21. Lemaitre F, Coquart JB, Chavallard F, Castres I, Mucci P, Costalat G, et al. Effect of additional respiratory muscle endurance training in young well-trained swimmers. J Sports Sci Med 2013;12:630-8.

22. Walker DJ, Ertl T, Walterspacher S, Schlager D, Roecker K, Windisch W, et al. Respiratory muscle function during a sixweek period of normocapnic hyperpnoea training. Respir Physiol Neurobiol 2013;188:208-13.

23. Bodden JG, Needham RA, Chockalingam N. The effect of an intervention program on functional movement screen test scores in mixed martial arts athletes. J Strength Cond Res 2015;29: 219-25.

24. Chapman RF, Laymon AS, Arnold T. Functional movement scores and longitudinal performance outcomes in elite track and field athletes. Int J Sports Physiol Perform 2014;9:203-11.

25. Chorba RS, Chorba DJ, Bouillon LE, Overmyer CA, Landis JA. Use of a functional movement screening tool to determine injury risk in female collegiate athletes. N Am J Sports Phys Ther 2010;5:47-54.

26. Bradley H, Esformes J. Breathing pattern disorders and functional movement. Int J Sports Phys Ther 2014;9:28-39. 